\title{
Splitting procedures for the Mixed Capacitated Arc Routing Problem under Time restrictions with Intermediate Facilities
}

\author{
Elias J. Willemse $e^{\mathrm{a}, 1, *}$, Johan W. Joubert ${ }^{\mathrm{a}, 2}$ \\ ${ }^{a}$ Center of Transport Development, Department of Industrial and Systems Engineering, University of Pretoria, South Africa, 0002
}

\begin{abstract}
This paper develops optimal and quick near-optimal splitting procedures for the Mixed Capacitated Arc Routing Problem under Time restrictions with Intermediate Facilities. Splitting procedures are a key component of giant tour-based solution methods for Arc Routing Problems. The optimal and near-optimal splitting procedures are tested within a multi-start constructive heuristic, and a fixed execution-time limit is imposed. Results on benchmark instances show that the constructive-heuristic linked with the new optimal splitting algorithm performs better than the near-optimal versions.
\end{abstract}

Keywords: Waste collection; Capacitated Arc Routing Problem; Intermediate Facilities; Mixed network; Splitting procedures.

\section{Introduction}

The Capacitated Arc Routing Problem under Time restrictions with Intermediate Facilities (CARPTIF), first proposed by Ghiani et al. [6] $]^{3}$, is a variant of the classical Capacitated Arc Routing Problem (CARP) and models residential waste collection. On a mixed network, with one and two-way streets in the case of waste collection, the problem is termed the Mixed CARPTIF (MCARPTIF), first proposed by Willemse and Joubert [13]. The problem considers a graph $\boldsymbol{G}=(\boldsymbol{V}, \boldsymbol{E} \cup \boldsymbol{A})$, where $\boldsymbol{V}$ represents the set of vertices, $\boldsymbol{E}$ represents the set of undirected edges that may be traversed in both directions, and $\boldsymbol{A}$ represents the set of arcs that can only be traversed in one direction. For waste collection, $\boldsymbol{V}$ corresponds to road intersections and dead-ends, while $\boldsymbol{E}$ and $\boldsymbol{A}$ model road segments between vertices. A subset of required edges and $\operatorname{arcs}, \boldsymbol{E}_{r} \subseteq \boldsymbol{E}$ and $\boldsymbol{A}_{r} \subseteq \boldsymbol{A}$, must be serviced by a fleet of $K$ homogeneous vehicles with limited capacity, $Q$, that are based at the depot vertex, $v_{1}$. The fleet size $K$ can be either fixed, left as a decision variable or treated as unlimited. Vehicles are allowed to unload their waste at any Intermediate Facility (IF) at a cost of $\lambda$, and resume their collection routes. At the end of its route a vehicle must first visit an IF before returning to the depot. The set of IFs is modelled in $\boldsymbol{G}$ as $\boldsymbol{\Gamma}$, where $\boldsymbol{\Gamma} \subset \boldsymbol{V}$. The sum of demand on each sub-trip between IF visits may not exceed $Q$, and unless $v_{1} \in \Gamma$, a vehicle has to visit an IF before returning to the depot. Lastly, a route length or time restriction of $L$ is imposed on each vehicle route. For a comprehensive review of

\footnotetext{
${ }^{*}$ Corresponding author

Email addresses: ejwillemse@gmail .com (Elias J. Willemse), johan.joubert@up.ac.za (Johan W. Joubert)

${ }^{1}$ Tel: +27 12420 3443; Fax: +27 123625103 (E.J. Willemse)

${ }^{2}$ Tel: +27 12420 2843; Fax: +27 123625103 (J.W. Joubert)

${ }^{3}$ The problem is also referred as the Arc Routing Problem with Intermediate Facilities under Capacity and Length Restrictions (CLARTPIF),
}

the CARP and other Arc Routing Problems we refer the reader to Corberán and Laporte [3] and Corberán and Prins [4].

Since the CARP and all its extensions are $\mathcal{N} \mathcal{P}$-hard the most effective methods for solving the problems are based on heuristics and metaheuristics, many of which employ giant tour approaches that rely on tour splitting procedures [10]. Splitting procedures take as input a giant tour and partitions the tour into feasible vehicle routes. In this paper we present optimal and heuristic splitting procedures for the MCARPTIF that can be used in giant tour approaches for the problem. The structure of our optimal splitting procedure provides a substantial improvement in efficiency over the existing CAPRTIF version that we adapted for mixed networks. Fast, near-optimal splitting procedures are also presented. The optimal and near-optimal procedures were tested in a multi-start Route-First-Cluster-Second heuristic on large MCARPTIF instances. Tight time-limits were imposed to reduce the number of starts of the slower, optimal procedures compared to the faster near-optimal procedures. Even with less starts, the Route-First-Cluster-Second heuristic linked with our efficient optimal splitting procedure performed better than the near-optimal splitting versions.

The following is an outline of the remainder of the paper. In the next section we review current splitting procedures for the CARP and a few of its extensions. In Section 3 we present the algorithm notation and detailed descriptions of our splitting procedures. In Section 4 we report on computational experiments, focusing on the execution times of the procedures and the difference in partition costs between optimal and nearoptimal splitting. We then compare the performance of the different procedures within a multi-start Route-First-ClusterSecond heuristic. The paper concludes in Section 5 with a summary of our main findings and suggestions for future research opportunities. 


\section{Current splitting procedures for the CARP and CAPRTIF}

The first optimal splitting procedure for the CARP was developed by Ulusoy [12] as part of a Route-First-Cluster-Second constructive heuristic. The heuristic is similar to the one of Beasley [1] for the Vehicle Routing Problem. First, edge demands are ignored and a giant tour is constructed servicing all the required edges in $\boldsymbol{G}$. In the second phase, an auxiliary Directed Acyclic Graph (DAG) is constructed whose arcs represent feasible sub-tours of the giant tour, with respect to demand of the sub-tour and vehicle capacity. The DAG is constructed in such a way that the shortest path through the graph gives the optimal partition of the giant tour into feasible vehicle routes. The shortest path can be calculated using any shortest path algorithm. The splitting procedure consists of constructing the DAG, calculating the shortest path through the graph, and decoding the shortest path to retrieve the optimal giant tour partitions. Lacomme et al. [8] and Belenguer et al. [2] develop multi-start Route-First-Cluster-Second heuristics for the CARP and Mixed CARP (MCARP), respectively, whereby different giant tours are constructed and partitioned, and the best returned as the final solution.

Ghiani et al. [7] develop a splitting procedure, similar to CARP versions, for the Capacitated Arc Routing Problem with Intermediate Facilities (CARPIF). Their procedure calculates the optimal placement of Intermediate Facility (IF) visits within a route. The problem allows inter-route offloads so that collected demand between IF visits never exceeds vehicle capacity, but it does not impose route duration limits. As such, a solution always consists of only one route. When a route duration limit is imposed the problem generalises to the CAPRTIF. To solve the problem Ghiani et al. [6] develop a splitting procedure that constructs two DAGs. The first consists of multiple source and destination vertices, each representing a start- and end-edge of a sub-tour in the giant tour. Shortest paths through the DAG between the sources and destinations represent the optimal placement of IFs in all possible sub-tours. The shortest path costs, calculated using a shortest path algorithm, are then used to construct a second DAG whose shortest path represents the optimal partition of the giant tour into vehicle routes. The optimal placement of IFs in each route is traced back to the shortest paths in the first DAG. The splitting procedure of Ghiani et al. [6] can be applied as-is to giant tours on mixed networks. A solution for the MCARPTIF can thus be obtained by combining the Route-First phase of Belenguer et al. [2] for the MCARP to construct a giant tour on a mixed network, and then applying the CARPTIF splitting procedure of Ghiani et al. [6] for the Cluster-Second phase.

To improve the efficiency of splitting procedures Lacomme et al. [8] develop a compact procedure for the CARP that does not explicitly construct the DAG. Instead, the shortest path through the DAG is directly calculated when scanning sub-tours for their feasibility with respect to vehicle capacity limits. Their version also accounts for a secondary objective of minimising fleet size. The compact version is exclusively used in Memetic Algorithms for the CARP [8, 9, 11] and MCARP [2], which are currently some of the most effective solution methods for the problems. Memetic Algorithms are metaheuristics based on genetic algorithms enhanced with local search procedures. Chromosomes are encoded as giant tours and an optimal splitting procedure is used to determine chromosome fitness each time a new chromosome is evaluated. An efficient splitting procedure is critical for the applications since chromosome evaluation occurs tens of thousands of times during the MA's execution.

In this paper we extended the compact splitting version of Lacomme et al. [8] to the MCARPIF. We then further extended this version to deal with the MCARPTIF and show that it provides a substantial improvement in efficiency over the version of Ghiani et al. [6]. We also developed two quick heuristic splitting procedures, one that greedily inserts IF visits into sub-tours and then calculates the route partitions and a second that employs a next-fit bin-packing procedure.

\section{New splitting procedures}

Before presenting our splitting procedures we first describe the graph transformation of $\boldsymbol{G}$ and introduce the basic algorithm notation. Consistent with Belenguer et al. [2] and Lacomme et al. [8], the graph $\boldsymbol{G}$ is transformed into a fully directed graph, $\boldsymbol{G}^{*}=\left(\boldsymbol{V}, \boldsymbol{A}^{*}\right)$. CARPTIF splitting procedures can then be used as-is on the MCARPTIF, and the other way around. Required arcs, $\boldsymbol{A}_{r}$, and edges, $\boldsymbol{E}_{r}$, of $\boldsymbol{G}$ correspond in $\boldsymbol{G}^{*}$ to a subset $\boldsymbol{R} \subseteq \boldsymbol{A}^{*}$ of required arcs. Each arc, $u \in \boldsymbol{R}$, has a demand, $q(u)$, a collection cost, $w(u)$, and a pointer, $\operatorname{inv}(u)$, to the arc between the same vertices but in the opposite direction. Each required arc in the original graph, $\boldsymbol{G}$, is coded in $\boldsymbol{R}$ by one arc, $u$, with $\operatorname{inv}(u)=0$, while each required edge is encoded as two opposite arcs, $u$ and $v$, such that $\operatorname{inv}(u)=v$ and $\operatorname{inv}(v)=u$. The depot is modelled by including in $\boldsymbol{A}^{*}$ a fictitious loop, $\sigma$, with zero deadheading and service cost. Similarly, the set of IFs are modelled in $\boldsymbol{A}^{*}$ as a set of dummy arcs, $\boldsymbol{I}$, such that each IF in $\boldsymbol{\Gamma}$ is modelled as a fictitious loop, $\Phi_{i} \in \boldsymbol{I}$, and $\Phi_{i}$ also has zero deadheading and service cost. The cost of the shortest path from arc $u$ to arc $v$, which excludes the costs of deadheading $u$ and $v$, is given by $D(u, v)$, which is pre-calculated for all arcs in $\boldsymbol{A}^{*}$. Shortest paths can be efficiently calculated using a modified version of Dijkstra's algorithm, and may also incorporate forbidden turns and turn-penalties [8]. The best IF to visit after servicing arc $u$ and before servicing $\operatorname{arc} v$ can be pre-calculated using

$$
\begin{aligned}
& \Phi^{*}(u, v)=\arg \min \{D(u, k)+D(k, v): k \in \boldsymbol{I}\}, \\
& \mu^{*}(u, v)=D\left(u, \Phi^{*}(u, v)\right)+D\left(\Phi^{*}(u, v), v\right)+\lambda,
\end{aligned}
$$

where $\Phi^{*}(u, v)$ gives the best IF to visit, and $\mu^{*}(u, v)$ gives the cost of the visit, including the unloading cost, $\lambda$, and deadheading costs. We denote by $\boldsymbol{S}$ the giant tour to be partitioned, which consists of a list of tasks, $\left[S_{1}, \ldots, S_{|S|}\right]$. It is assumed that the shortest path is always followed between consecutive tasks and only arcs in $\boldsymbol{R}$ are included in $\boldsymbol{S}$, thus it contains no depot or IF dummy arcs as these are implicitly accounted for by the splitting procedures. Sub-tours in $\boldsymbol{S}$ are denoted as $\boldsymbol{S}_{i \rightarrow j}=\left[S_{i}, \ldots, S_{j}\right]$ where $1 \leq i<j \leq n$ and $n=|\boldsymbol{S}|$. A 
single MCARPIF or MCAPRTIF route is a list of tasks that always starts with the dummy depot task, ends with a dummy IF and depot task, and may include inter-route IF visits. The list of tasks between dummy arcs represent subtrips and the load collected on a subtrip may not exceed $Q$. For the MCAPRTIF, the total cost of a route, including all task service costs, deadheading costs between tasks and IF visit costs, may not exceed $L$.

\subsection{Splitting procedures for the MCARPIF}

The first splitting procedure that we present is an MCARPIF adaption of the CARP procedure developed by Lacomme et al. [8]. Recall that splitting procedures use $S$ to construct an auxiliary DAG, $\boldsymbol{H}$, in such a way that its shortest path represents the optimal giant tour partition. For the CARPIF, Ghiani et al. [7] construct $\boldsymbol{H}$ by including a vertex for each feasible subtour $S_{i \rightarrow j}$ with respect to vehicle capacity. Vertices representing consecutive sub-tours $\boldsymbol{S}_{i \rightarrow j}$ and $\boldsymbol{S}_{j+1 \rightarrow k}$ are then linked with arcs. A source vertex, linked to vertices representing $\boldsymbol{S}_{1 \rightarrow j}$, and a sink vertex, linked to vertices representing $\boldsymbol{S}_{k \rightarrow|\boldsymbol{S}|}$, are also included in $\boldsymbol{H}$ and the shortest path from the source to the sink represents the optimal partition. Using this approach the DAG consists of at most $\frac{n(n+1)}{2}$ vertices and $\frac{n(n+1)(n-1)}{6}+2 n$ arcs. Bellman's algorithm [5] can then compute the shortest path through $\boldsymbol{H}$ in $\boldsymbol{O}\left(n^{3}\right)$. For the CARP, Lacomme et al. [8] construct $\boldsymbol{H}$ so that it consists of exactly $n+1$ vertices and at most $\frac{n(n+1)}{2} \operatorname{arcs}$ where each arc, instead of vertices, represents a feasible subtour. Computing the shortest path through $\boldsymbol{H}$ then takes $O\left(n^{2}\right)$. For a detailed illustration of the method we refer the reader to Appendix A.

To improve the efficiency of split for the CARP, Lacomme et al. [8] develop a compact version that does not explicitly construct $\mathbf{H}$. Algorithm 1 shows a similar procedure, called Efficient-IF-Split, that we developed for the MCARPIF. It directly calculates the optimal partition and further minimises the number of subtrips as a second objective. Modifications to the CARP procedure of Lacomme et al. [8] are contained in Lines 1, and 9-19, with the CARP calculations shown as comments $(/ /)$ in Lines 2, 13, 16 and 19. Three labels are used by the procedure. The first, $\boldsymbol{N}_{i}$, represents the cost of the shortest path from vertex 0 to $i$ in $\boldsymbol{H}$, the second, $\boldsymbol{\Pi}_{i}$, represents the number of sub-trips in the same shortest path, and the third, $\boldsymbol{P}_{i}$, represents the predecessor vertex of $i$ on this path and thus stores the resulting optimal placement of IFs in $S$. Note that both $N$ and $\boldsymbol{P}$ are indexed from 0 . The depot and final IF and depot visit on a feasible route are implicitly accounted for by the algorithm. By increasing $i$ and $j$ the procedure successively scans sub-tours for capacity violations. When the feasible sub-tour $S_{i \rightarrow j}$ is found the optimal partition for the partial giant tour ending at $j$ is updated. In the worst case a total of $\frac{n(n+1)}{2}$ sub-tours can be evaluated, giving the algorithm a running time of $O\left(n^{2}\right)$. The actual running time of the algorithm is reduced as only feasible sub-tours that meet the capacity constraint are evaluated.

\subsection{Splitting procedures for the MCAPRTIF}

For the MCAPRTIF the splitting procedure has to simultaneously determine the optimal IF partitions, resulting from

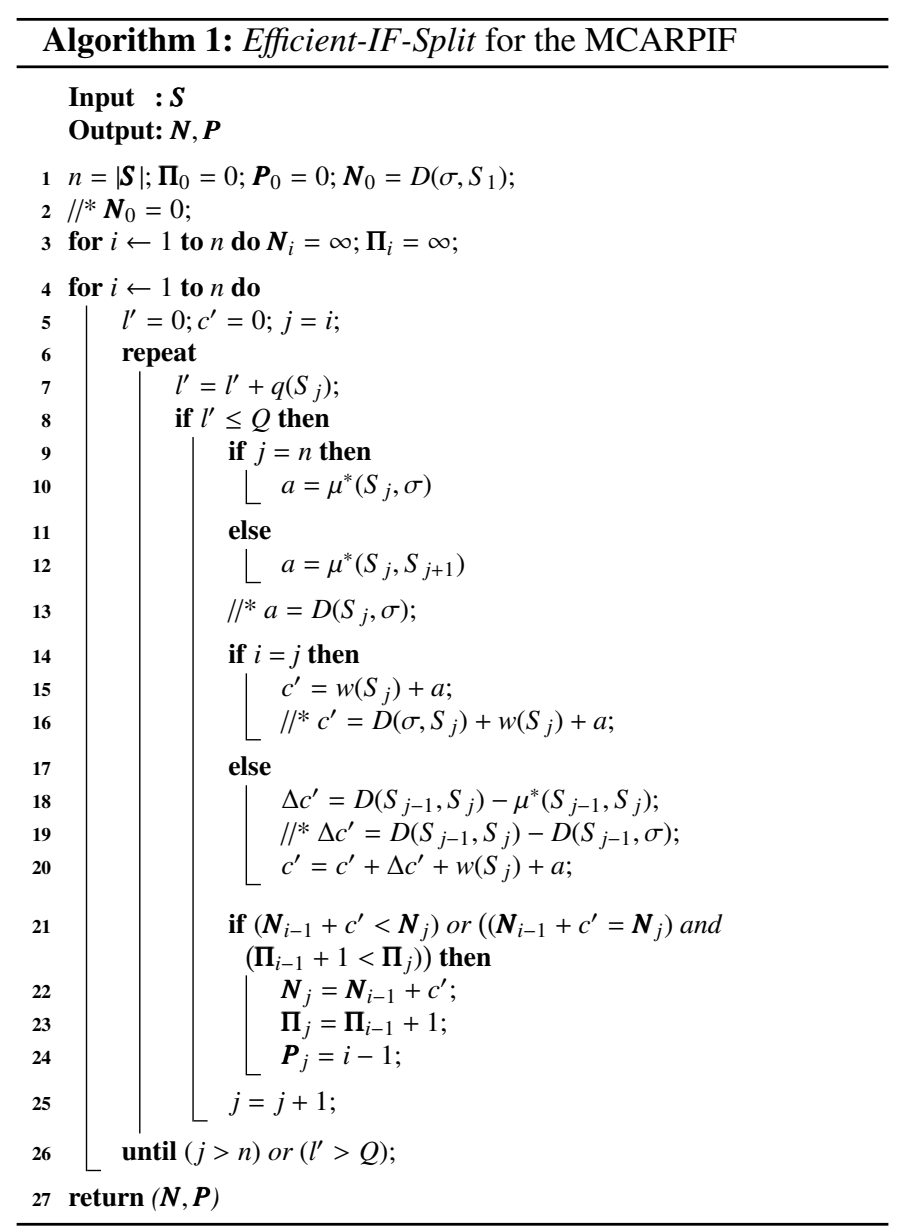

*Original calculations of Lacomme et al. [8] for the CARP. 
the vehicle capacity limit $Q$, and the optimal route partitions, resulting from the route time restriction, $L$. Ghiani et al. [6] extend the method of Ghiani et al. [7] and explicitly construct two DAGs for this purpose. Efficient-IF-Split can be used in the same way. First, it calculates the optimal IF partitions for all the sub-tours in $S$. The cost of the partitioned sub-tours are then used to construct the second DAG, and the shortest path through it represents the optimal route partitions. For a detailed illustration of the method we refer the reader to Appendix A.

Similar to Efficient-IF-Split, $\boldsymbol{H}$ and $\boldsymbol{H}^{\prime}$ need not be explicitly constructed. Algorithm 2 shows a compact splitting version for the MCAPRTIF that directly calculates the optimal partitions for subtrips and routes. We refer to this version as EfficientSplit and to the version that calculates the optimal IF and route partitions in two-phases as Two-Phase-Split. Algorithm 2 minimises the total number of routes as the primary objective, and partition cost as secondary. For the IF partitions, two labels are used for each vertex $i$ in $\boldsymbol{H}$. The first, $\boldsymbol{N}_{i, j}$, where $i \geq j$, represents the cost of the shortest path from vertex $i$ to $j$ in $\boldsymbol{H}$, and the second, $\boldsymbol{P}_{i, j}$, where $i \geq j$, represents the predecessor vertex of $j$ on this path back to $i$ and thus stores the resulting optimal placement of IFs in sub-tours of $\boldsymbol{S}$. Two more labels are used for the optimal route partitions. The first, $\boldsymbol{N}_{i}^{\prime}$, represents the cost of the shortest path from vertex 0 to $i$ in $\boldsymbol{H}^{\prime}$, and the second, $\boldsymbol{P}_{i}^{\prime}$, represents the predecessor vertex of $i$ on this path and thus stores the resulting optimal route partitions of $\boldsymbol{S}$. Since the MCAPRTIF has multiple routes, the partitioning algorithm is further extended by including $\Pi_{i}$, which represents the number of routes required from vertex 0 to $i$ in $\boldsymbol{H}^{\prime}$. By increasing $i$ and $j$ the procedure successively scans sub-tours for capacity violations. When the feasible sub-tour $\boldsymbol{S}_{i \rightarrow j}$ is found the optimal partitions for all sub-tours starting at $k \in\{1, \ldots, i\}$ and ending at $j$ are updated. In the worst case, a total of $\frac{n(n+1)(n+2)}{6}$ sub-tours are evaluated for the optimal IF partitions, giving the algorithm a running time of $O\left(n^{3}\right)$. The actual running time of Algorithm 2 is reduced as only feasible sub-tours, adhering to the route time restriction, are optimally partitioned with IF visits. When $i=j$ the optimal partition of all sub-tours from $k \in\{1, \ldots, j\}$ to $j$ have been calculated. If $\boldsymbol{S}_{k \rightarrow j}$ then exceeds $L$, longer sub-tours starting at $k$ will also exceed $L$ and they need not be updated with IF partitions in subsequent iterations. This significantly reduces the number of sub-tours that have to be updated each time $i$ and $j$ are incremented.

\subsection{Heuristic splitting procedures for the MCAPRTIF}

The last two splitting procedures that we developed for the MCAPRTIF are heuristic in nature and thus do not guarantee an optimal partition. The first is a straight forward Next-Fit binpacking type procedure that starts with a route consisting of the starting depot task. Starting with the first task in $S$, tasks are progressively added in sequence to the route. If a task cannot be added without exceeding $Q$, an IF visit is included before the task. If a task cannot be added to the route without exceeding $L$, including the cost of going from the task to the nearest IF and depot, the route is closed and the task is added to a new route. The procedure, which we call Simple-Split, runs in $O(n)$.

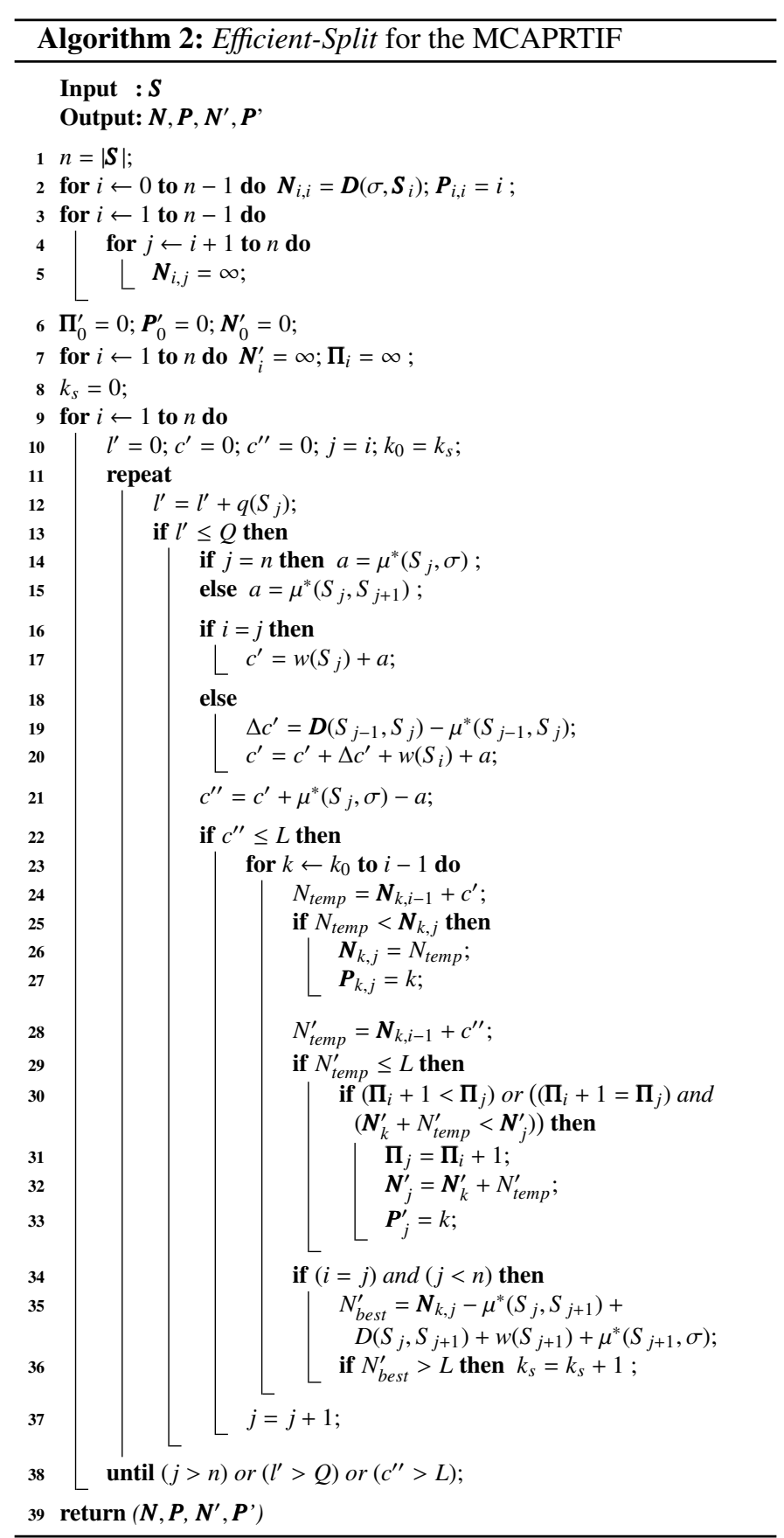


The second heuristic procedure that we developed, called Heuristic-Split, is shown in Algorithm 3. It is an extension of

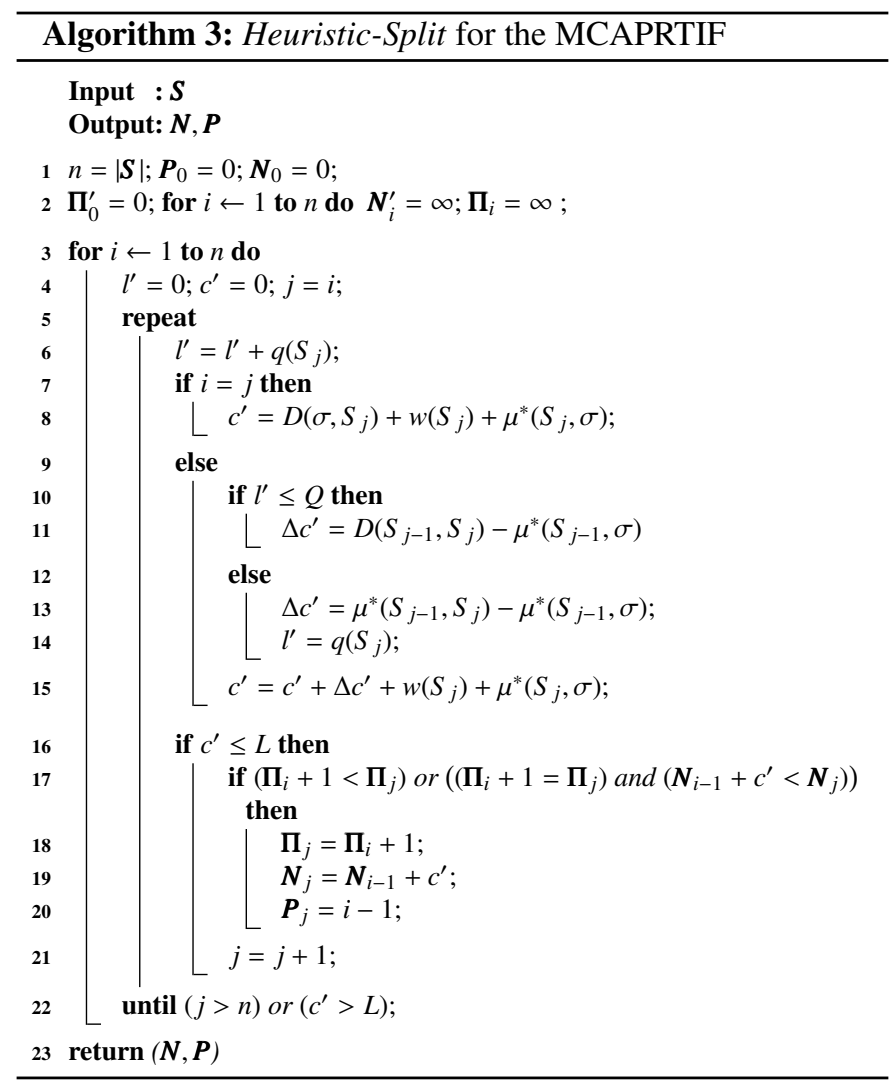

Efficient-IF-Split whereby IF visits are included in routes greedily instead of optimally. When evaluating progressively longer sub-tours, IF visits are inserted the moment that the load of a sub-tour since the last IF insertion exceeds vehicle capacity. The optimal placement of IFs is not calculated for each subtour, reducing the computational complexity of the algorithm to $O\left(n^{2}\right)$ while still ensuring that it produces feasible routes with respect to $Q$ and $L$. After partitioning $\boldsymbol{S}$, the IF partitions for each of the resulting routes can be improved using Efficient-IFSplit while maintaining the overall complexity of $O\left(n^{2}\right)$.

\section{Computational results}

For the computational tests we analysed and compared the efficiency of Two-Phase-Split, Efficient-Split, Simple-Split and Heuristic-Split for the MCAPRTIF. Efficiency was measured as the time taken by each procedure to partition a giant tour. We then compared the number of routes and cost of the giant tour partitions from each procedure. Heuristic-Split was always linked with Efficien-IF-Split as a post-partition procedure to improve the IF placements in each route. The aim was to determine if Simple-Split or Heuristic-Split could be potentially used as a substitute for Two-Phase-Split and Efficient-Split in giant tour solution methods when solving large scale instances.

Two sets of MCAPRTIF benchmark sets developed in [14] were used for our tests. The first set, $L p r-I F$, is based on the $l p r$
MCARP set of Belenguer et al. [2], and the second set, referred to as Cen-IF, is based on actual road networks and contains some of the largest arc routing instances currently available. All heuristics were programmed in Python version 2.7, with critical procedures optimised using Cython version 0.17.1. Computational experiments were run on a PC with a $2.5 \mathrm{Ghz}$ Intel Core i5 processor and with $8 \mathrm{~GB}$ memory. For more information on the benchmark instances, including download-links, we refer the reader to DATA IN BRIEF.

To compare the efficiency of the procedures thirty giant tours were constructed using Path-Scanning-Random-Link developed by Belenguer et al. [2] for the MCARP and relaxing the capacity limit and route time restriction, as proposed by the authors. The giant tours were then partitioned using one of the four splitting procedures with the primary objective to minimise the number of required routes, and secondly to minimise the partition cost. Figure 1 shows the CPU time taken by each procedure to partition the giant tours as a function of the size of the tours, $n$. Giant tours for Cen-IF instances are of sizes 1012, 2519 and

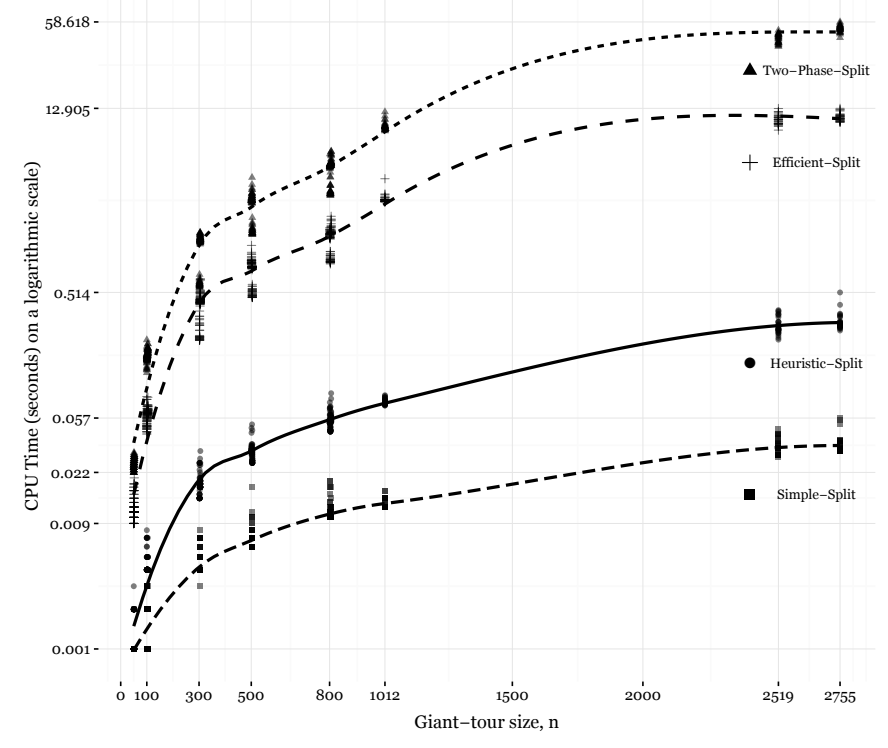

Figure 1: Scatter plots and trend lines for giant tour size, $n$, versus partitioning time (in seconds, on a logarithmic scale) of four MCAPRTIF splitting procedures on 30 different giant tours per instance.

2755. All other points in the figure are for the Lpr-IF instances. All four splitting procedures show polynomial growth in their execution times as a factor of $n$. Simple-Split was extremely efficient, since it runs in $O(n)$, splitting the Cen-IF tours with over 2500 tasks in less 0.05 seconds. Heuristic-Split was also efficient, taking less than 0.5 seconds to partition the Cen-IF tours. Owing to their $O\left(n^{3}\right)$ complexity, Two-Phase-Split and Efficient-Split took longer to partition tours. On all instances, Efficient-Split was substantially quicker than Two-Phase-Split, though on small instances with $n \leq 104$ the difference in performance may not be practically significant, with both procedures taking less than 0.5 seconds per partition. Two-Phase-Split took close to 60 seconds on the largest Cen-IF instances, whereas 
Efficient-Split took only 12 seconds.

Next we compared the solution quality of the partitioning procedures over the thirty Path-Scanning-Random-Link giant tours per instance. Since Two-Phase-Split and Efficient-Split produce the same optimal partitions their partition costs and resulting number of routes were used as a target. For the primary objective, to minimise the number of routes resulting from the partition, both Simple-Split and Heuristic-Split matched TwoPhase-Split on more than 79 of the 90 Cen-IF and on 431 of the 450 Lpr-IF giant tours. On the remaining tours, the nearoptimal procedures required at most one additional route. To see how close the suboptimal partitions were to the optimal partitions, cost wise, the cost gap between the partitions was measured as $Z_{g a p}=\frac{Z-Z^{*}}{Z^{*}}$ where $Z$ is the cost of the final partition of the giant tour, partitioned using Simple-Split or Heuristic-Split, and $Z^{*}$ is the cost of the optimal partition, as calculated through Efficient-Split. Results per problem instance are shown in Figure 2 where each instance is labelled according to its giant tour size, $n$. Except for two instances, the partitions produced by

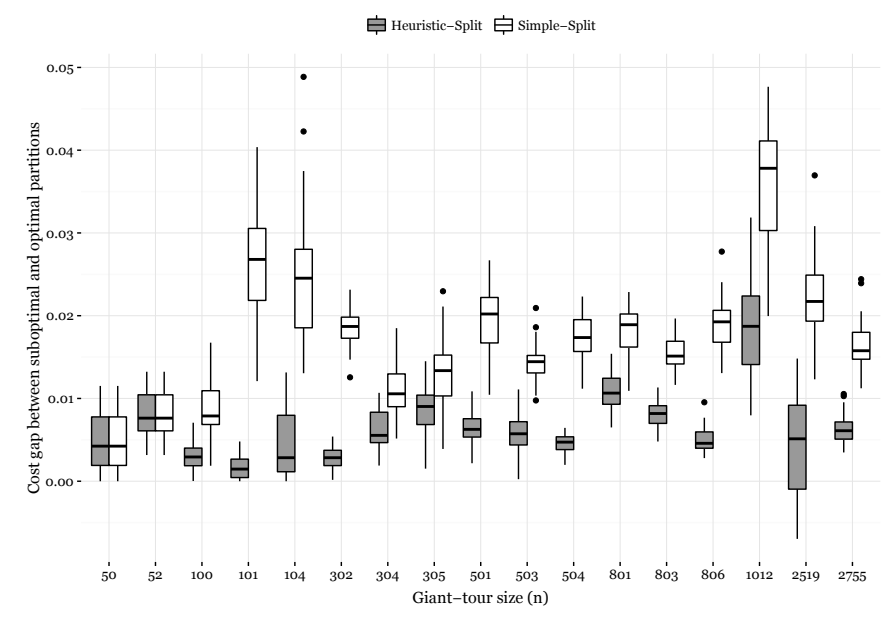

Figure 2: Box-and-Whisker plot for the cost gap, $Z_{\text {gap }}$, between the partitions of Simple-Split and Heuristic-Split, and the optimal partitions of Efficient-Split.

Heuristic-Split were very close to optimal with an average cost gap of less than $1 \%$. The cost gap range (maximum gap minus mininum gap) of the procedure is also small, being less than or close to $2 \%$, which shows that it consistently produced nearoptimal partitions. Negative cost gaps were observed for the procedure on some of the giant tours for Cen-IF-c. These were tours where Heuristic-Split partitions required an extra route over the optimal partitions, and the extra route resulted in a lower solution cost. Since minimising the number of routes was our primary objective, these partitions are suboptimal. SimpleSplit matched Heuristic-Split on the two smallest giant tour instances. On all other instances its cost gap was larger, which was expected given its simple structure. The cost gap range of Simple-Split was also larger than Heuristic-Split, showing that its performance was not as consistent.

Our results show that as a factor of giant tour size, the execution time of Heuristic-Split is an order of magnitude lower to that of Efficient-Split, whose computation time is an order of magnitude lower than Two-Phase-Split (Figure 1). Furthermore, Heuristic-Split consistently produced near-optimal partitions, whereas Simple-Split resulted in higher cost partitions and its performance varied per instance (Figure 2). In time limited applications, methods linked with Simple-Split and HeuristicSplit may produce better results than Two-Phase-Split and EfficientSplit since their higher efficiency will allow the methods to evaluate and split more giant tours.

To test the hypothesis the following experiment was conducted using a multi-start Route-First-Cluster-Second constructive heuristic. Path-Scanning-Random-Link (PSRL) was again used for the Route-First phase and different splitting procedures were used for the Cluster-Second phase. Giant tours, representing different starts, were generated with PSRL and partitioned by the splitting procedure until an execution time-limit of 60 seconds was reached. The best partitioned solution from all the starts was then returned as the final solution. This was repeated for 30 experiments per instance and the mean number of routes, $\bar{K}$, and cost, $\bar{C}$, over the returned solutions were calculated. Summary results over the Lpr-IF and Cen-IF problem sets are shown in Table ??. Within the multi-start heuristic all the procedures performed the same in minimising the number of solution routes. The only difference in performance was in minimising solution cost. As expected, Efficient-Split performed better than Two-Phase-Split since it was able to make more starts in 60 seconds while still producing optimal partitions. On small instances the difference between Efficient-Split and Two-Phase-Split was less prominent. Heuristic-Split outperformed Two-Phase-Split on all the Cen-IF instances, and five of the fifteen Lpr-IF instances. Simple-Split had up to 30 and 800 times more starts than Efficient-Split and Heuristic-Split, respectively, on large Cen-IF and Lpr-IF instances, but could only match Efficient-Split on one and Heuristic-Split on three of the smallest Lpr-IF instances. Solution costs of HeuristicSplit are within one percent of Efficient-Split and it performed better on the Cen-IF-c instance. On all other instances EfficientSplit performed the best, making it the best constructive heuristic implementation on both small and large instances, with and without execution time-limits imposed.

For metaheuristics the effect of using Simple-Split or HeuristicSplit instead of Efficient-Split is difficult to analyse without embedding the procedures within a Memetic Algorithm (MA). The MA of Belenguer et al. [2] terminates after 26000 to 40000 evaluations, or when a one hour execution time limit is reached. If a similar time limit is imposed for the Cen-IF instances, and ignoring time required by the MA to perform other functions, such as Local-Search, Efficient-Split will be able to evaluate at most approximately 1350, 300, and 350 chromosomes for the three Cen-IF instances, respectively. Heuristic-Split will be able to evaluate at most 47000,12000 and 12500 chromosomes for the same problem instance. The impact of more chromosome evaluations based on near-optimal instead of optimal partitions can only be evaluated by implementing the procedures within an MA for the MCAPRTIF. 


\section{Conclusion}

Tests showed that Efficient-Split, developed in this paper, was significantly faster than Two-Phase-Split that was based on an existing splitting procedure for the CAPRTIF. On small instances the difference in performance between the two procedures was less prominent, highlighting the need for tests to be performed on more realistically sized instances. With this being, to our knowledge, the first formal study on splitting procedures for the MCAPRTIF, the next step will be to extend metaheuristics for the CARP and MCARP that rely on splitting procedures, such as Memetic Algorithms, at which point the effect of using Heuristic-Split instead of Efficient-Split under time-limits can be formally tested.

\section{Acknowledgements}

This work is based on the research supported in part by the National Research Foundation of South Africa (Grant Number 87749) and by the South African Department of Trade and Industry (Grant Number 96415).

\section{Appendix A. Supplementary material}

Supplementary material related to this article can be found online at http://ejwillemse.github.io/057/.

\section{References}

[1] Beasley, J. E. (1983). Route first-cluster second methods for vehicle routing. Omega, 11(4):403-408.
[2] Belenguer, J., Benavent, E., Lacomme, P., and Prins, C. (2006). Lower and upper bounds for the mixed capacitated arc routing problem. Computers $\mathcal{E}$ Operations Research, 33(12):3363-3383.

[3] Corberán, Á. and Laporte, G. (2015). Arc routing: problems, methods, and applications, volume 20. SIAM.

[4] Corberán, Á. and Prins, C. (2010). Recent results on arc routing problems: An annotated bibliography. Networks, 56(1):50-69.

[5] Cormen, T. H., Leiserson, C. E., and Rivest, R. L. (1990). Introduction to algorithms. MIT-Press.

[6] Ghiani, G., Guerriero, F., Laporte, G., and Musmanno, R. (2004). Tabu search heuristics for the arc routing problem with intermediate facilities under capacity and length restrictions. Journal of Mathematical Modelling and Algorithms, 3(3):209-223.

[7] Ghiani, G., Improta, G., and Laporte, G. (2001). The capacitated arc routing problem with intermediate facilities. Networks, 37(3):134-143.

[8] Lacomme, P., Prins, C., and Ramdane-Chérif, W. (2004). Competitive memetic algorithms for arc routing problems. Annals of Operations Research, 131(4):159-185.

[9] Liu, T., Jiang, Z., and Geng, N. (2013). A memetic algorithm with iterated local search for the capacitated arc routing problem. International Journal of Production Research, 51(10):3075-3084.

[10] Prins, C., Lacomme, P., and Prodhon, C. (2014). Order-first split-second methods for vehicle routing problems: A review. Transportation Research Part C: Emerging Technologies, 40:179-200.

[11] Tang, K., Mei, Y., and Yao, X. (2009). Memetic algorithm with extended neighborhood search for capacitated arc routing problems. Evolutionary Computation, IEEE Transactions on, 13(5):1151-1166.

[12] Ulusoy, G. (1985). The fleet size and mix problem for capacitated arc routing. European Journal of Operational Research, 22(3):329-337.

[13] Willemse, E. and Joubert W. (2016a). Constructive heuristics for the Mixed Capacity Arc Routing Problem under Time Restrictions with Intermediate Facilities. Computers $\mathcal{E}$ Operations Research, 68:30-62.

[14] Willemse, E. and Joubert W. (2016b). New benchmark sets for undirected and mixed capacitated arc routing problems under time restrictions with intermediate facilities. Submitted to Data in Brief. Available online from https://sites.google.com/site/wasteoptimisation/ capacitated-arc-routing-problems/working-papers. 


\title{
Supplementary material for: Splitting procedures for the Mixed Capacitated Arc Routing Problem under Time restrictions with Intermediate Facilities
}

\author{
Elias J. Willemse $e^{\mathrm{a}, 1, *}$, Johan W. Joubert ${ }^{\mathrm{a}, 2}$ \\ ${ }^{a}$ Center of Transport Development, Department of Industrial and Systems Engineering, University of Pretoria, South Africa, 0002
}

\begin{abstract}
This online companion contains detailed illustrations of splitting procedures from the manuscript Splitting procedures for the Mixed Capacitated Arc Routing Problem under Time restrictions with Intermediate Facilities, submitted for publication to Operations Research Letters.
\end{abstract}

\section{Splitting procedures for the MCARPIF}

In this section we show how the procedure of Lacomme et al. [2] can be extended for the MCARPIF. Figure 1 shows the partitioning of $\boldsymbol{S}=[a, b, c, d, e]$ into a feasible MCARPIF route with subtrips. The tour of Figure 1a is partitioned by building $\boldsymbol{H}=\left(\boldsymbol{V}^{\prime}, \boldsymbol{A}^{\prime}\right)$, with $n+1$ vertices, $\left\{v_{0}, \ldots, v_{n}\right\} \in \boldsymbol{V}^{\prime}$, indexed from 0 onward (Figure 1b). Each sub-tour, $\boldsymbol{S}_{i \rightarrow j}$, with load less than $Q$ represents a feasible subtrip and is modeled as $\left(v_{i-1}, v_{j}\right) \in \boldsymbol{A}^{\prime}$. Only feasible subtrips are modelled in $\boldsymbol{A}^{\prime}$. The cost of arc $\left(v_{i-1}, v_{j}\right)$ is equal to the subtrip cost, which includes the service cost of its tasks, the deadheading cost between tasks, and the cost of visiting an IF facility after $S_{j}$ and traveling to $S_{j+1}$ if $j<n$, or traveling from $S_{j}$ to an IF and then the depot if $j=n$. For example, the feasible sub-tour $\boldsymbol{S}_{2 \rightarrow 4}=[b, c, d]$ is modeled by the arc $\left(v_{1}, v_{4}\right)$ in $\boldsymbol{H}$, and its weight is 79 , which is the cost of servicing tasks $b, c$ and $d$, equaling $5+5+5=15$, plus the deadheading costs between tasks, equaling $20+20=40$, plus the cost of going to $\Phi_{2}$ and to task $e$, equaling $14+10=24$. The shortest path from vertex 0 to $n$ in $\boldsymbol{H}$ represents the optimal partition of $\boldsymbol{S}$ with IF visits (Figure 1c). Once $\boldsymbol{H}$ has been constructed, Bellman's algorithm for DAGs can efficiently compute the shortest path from vertex $v_{0}$ to $v_{n}$ in $\boldsymbol{H}$.

\section{Splitting procedures for the MCARPTIF}

To illustrate the Two-Phase-Split methods, the same giant tour in Figure 1a is used and a route time restriction of $L=70$ is imposed. It is further assumed that the shortest paths from the depot to tasks in $\boldsymbol{S}$, excluding task $a$, are through the respective IFs. For the first DAG, $\boldsymbol{H}$, each of the $\frac{n(n-1)}{2}$ sub-tours of $\boldsymbol{S}$ is included in $\boldsymbol{H}$, and each one is treated as a single route, starting at the depot, and ending with an IF and depot visit. Each sub-tour can be optimally partitioned with IF visits using Efficient-IF-Split. The result of the first phase on the example route is shown in Figure 2, in which the optimal partition of each sub-tour is calculated and given separately. Next, as shown in Figure 3, the algorithm uses the feasible sub-tours with total cost not exceeding $L$ to construct the second DAG, $\boldsymbol{H}^{\prime}=\left(\boldsymbol{V}^{\prime \prime}, \boldsymbol{A}^{\prime \prime}\right)$, with $n+1$ vertices, $\left\{v_{0}, \ldots, v_{n}\right\} \in \boldsymbol{V}^{\prime \prime}$, indexed from 0 onward (Figure 3a). Each sub-tour $\boldsymbol{S}_{i \rightarrow j}$ is modeled by one arc $\left(v_{i-1}^{\prime}, v_{j}^{\prime}\right)$ in $\boldsymbol{A}^{\prime \prime}$, weighted by the route's partitioned cost, given by the cost of the shortest path in $\boldsymbol{H}$ from $v_{i-1}$ to $v_{j}$. In Figure $3 \mathrm{a}$, arc $\left(v_{0}^{\prime}, v_{2}^{\prime}\right)$ in $\boldsymbol{H}^{\prime}$ models the route starting with task $S_{1}=a$ and ending with task $S_{2}=b$. Referring back to $\boldsymbol{H}$ (Figure 2), the optimal IF partition cost of this route is 67, which becomes the weight of arc $\left(v_{0}^{\prime}, v_{2}^{\prime}\right)$ in $\boldsymbol{H}^{\prime}$. Since the IF partitioned cost of the route is less than $L$, it is included in $\boldsymbol{H}^{\prime}$. The shortest path

\footnotetext{
${ }^{*}$ Corresponding author

Email addresses: ejwillemse@gmail.com (Elias J. Willemse), johan.joubert@up.ac.za (Johan W. Joubert)

${ }^{1}$ Tel: +27 124203443 ; Fax: +27 123625103 (E.J. Willemse)

${ }^{2}$ Tel: +27 12420 2843; Fax: +27 123625103 (J.W. Joubert)
} 


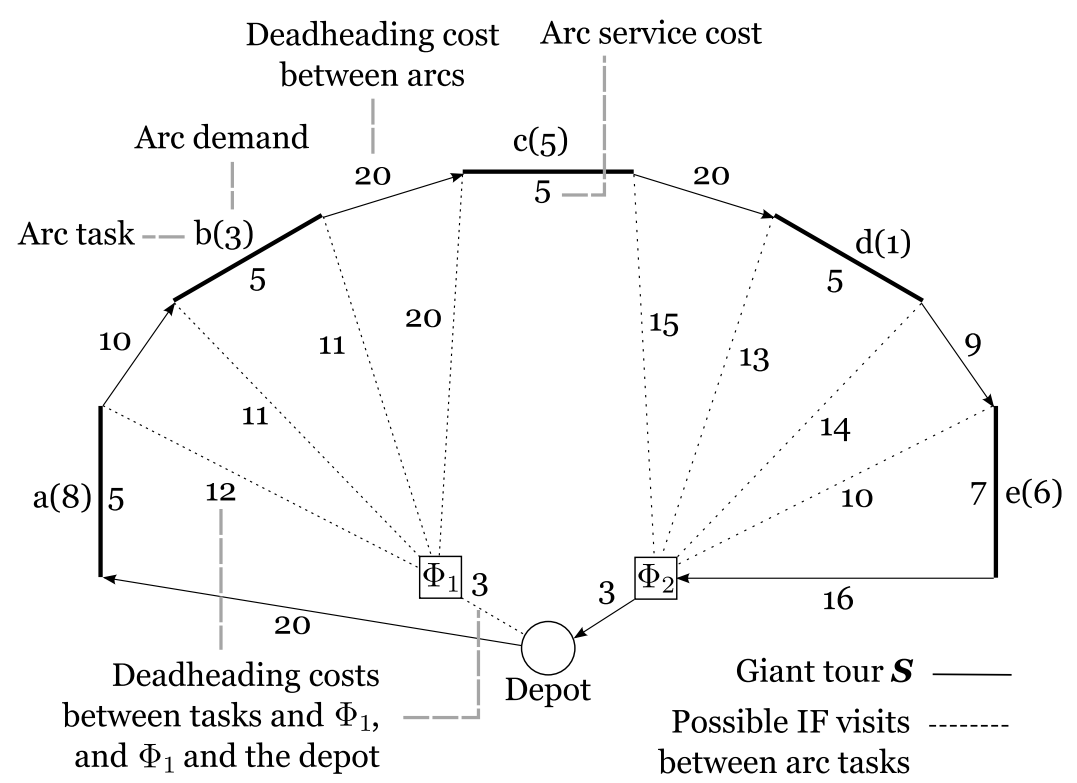

(a) Initial giant tour $S=[a, b, c, d, e]$ to be partitioned with IF visits. While not directly included in $\boldsymbol{S}$, it is assumed that the giant tour always starts at the depot, and ends with an IF and depot visit, as shown in the figure.

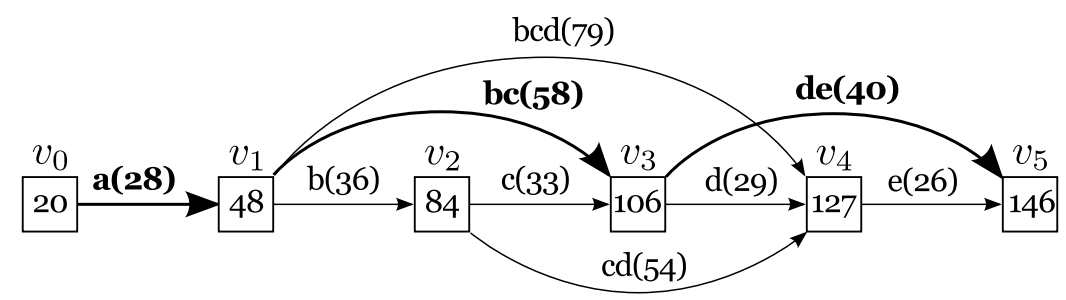

(b) Auxiliary Directed Acyclic Graph, $\boldsymbol{H}$, with the shortest path shown in bold representing the optimal IF partitions. Cost labels are shown in each vertex.

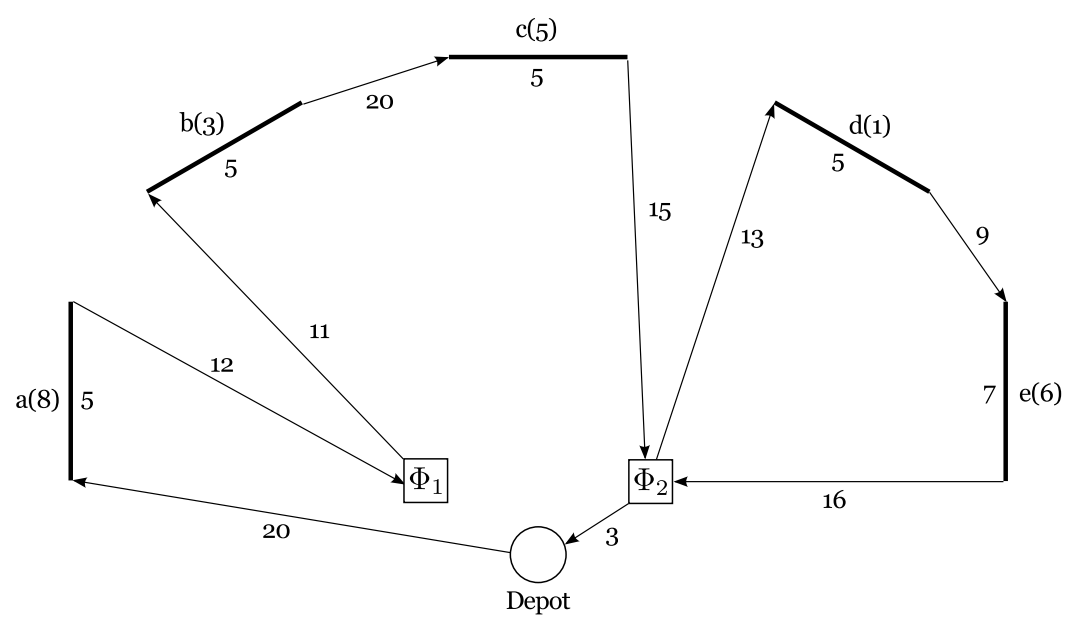

(c) Optimally partitioned route with IF visits, obtained through $\boldsymbol{H}$. The total cost of the partitioned route is 146, which is equal to the shortest path from $v_{0}$ to $v_{5}$ in $\boldsymbol{H}$.

Figure 1: Example of a splitting procedure for the MCARPIF, with vehicle capacity $Q=9$ and unloading $\operatorname{cost} \lambda=0$. 


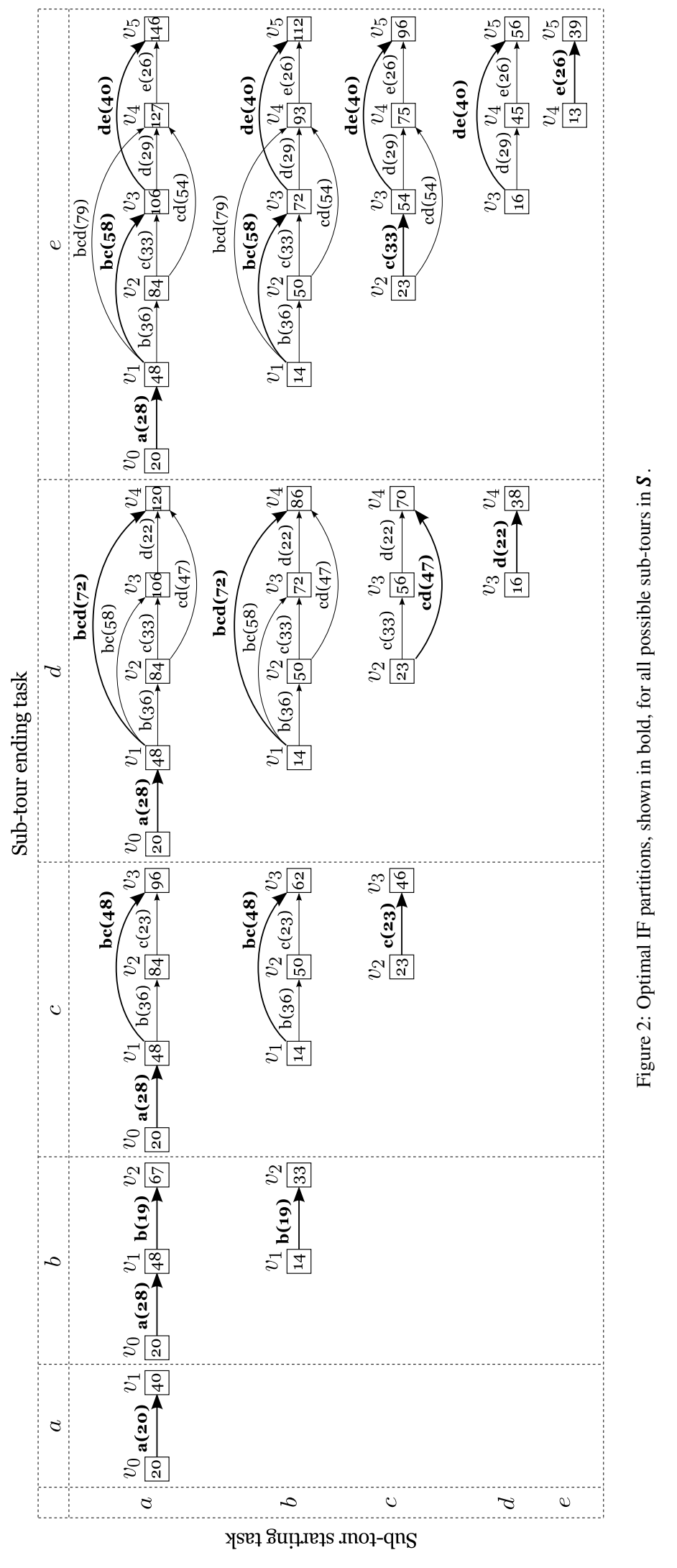


from vertex $v_{0}^{\prime}$ to $v_{n}^{\prime}$ in $\boldsymbol{H}^{\prime}$ represents the optimal route partition of $\boldsymbol{S}$, and the optimal IF visits for each route can be traced back to $\boldsymbol{H}$. The optimal route and IF partitions for the giant route in Figure 1a are shown in Figure $3 \mathrm{~b}$. When constructing $\boldsymbol{H}$, the optimal placement of IFs does not have to be calculated for all sub-tours. If the optimally

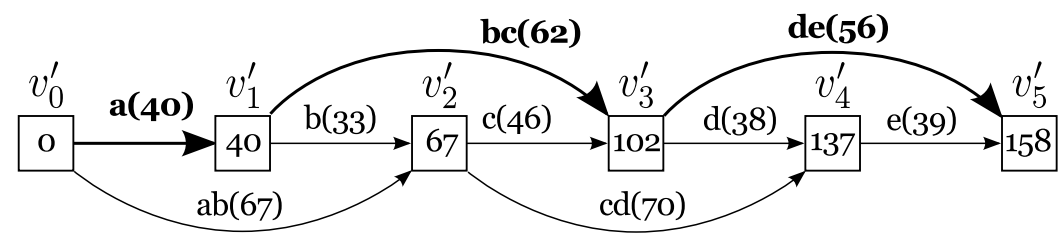

(a) Second auxiliary Directed Acyclic Graph, $\boldsymbol{H}^{\prime}$, constructed using $\boldsymbol{H}$ with the shortest path shown in bold representing the optimal route partitions. Cost labels are shown in each vertex.

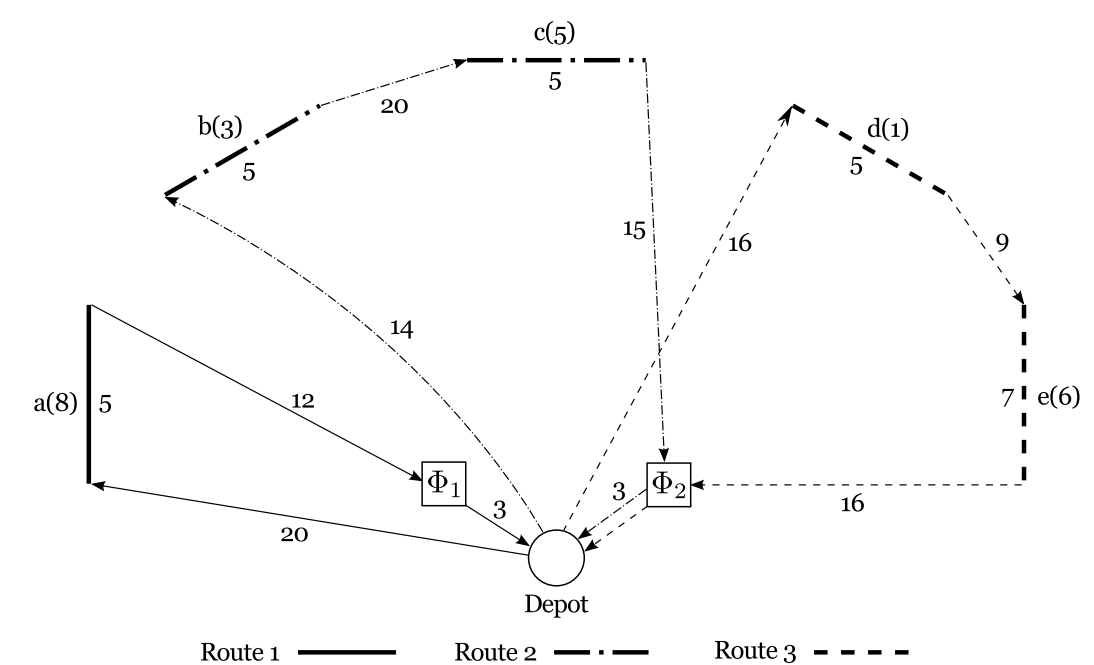

(b) Optimally partitioned giant tour with each route further partitioned with IF visits, as given by the shortest paths in $\boldsymbol{H}$ and $\boldsymbol{H}^{\prime}$.

Figure 3: Example of a splitting procedure for the MCLARPIF, with vehicle capacity $Q=9$, unloading cost $\lambda=0$ and route time restriction $L=70$.

partitioned sub-tour $\boldsymbol{S}_{i \rightarrow j}$ exceeds $L$, then all the partitioned sub-tours $\boldsymbol{S}_{i \rightarrow j+k}$, where $0<k \leq n-j$, will also exceed $L$ and can thus be skipped in the construction of $\boldsymbol{H}$.

\section{References}

[1] Belenguer, J., Benavent, E., Lacomme, P., and Prins, C. (2006). Lower and upper bounds for the mixed capacitated arc routing problem. Computers $\mathcal{E}$ Operations Research, 33(12):3363-3383

[2] Lacomme, P., Prins, C., and Ramdane-Chérif, W. (2004). Competitive memetic algorithms for arc routing problems. Annals of Operations Research, 131(4):159-185. 